\title{
Initial dual oral combination therapy in pulmonary arterial hypertension
}

\author{
Olivier Sitbon ${ }^{1,2,3}$, Caroline Sattler ${ }^{1,2,3}$, Laurent Bertoletti ${ }^{4,5}$, Laurent Savale ${ }^{1,2,3}$, \\ Vincent Cottin ${ }^{6}$, Xavier Jaïs ${ }^{1,2,3}$, Pascal De Groote ${ }^{7}$, Ari Chaouat ${ }^{8,9}$, \\ Céline Chabannes $^{10}$, Emmanuel Bergot $^{11}$, Hélène Bouvaist ${ }^{12}$, Claire Dauphin $^{13}$, \\ Arnaud Bourdin ${ }^{14}$, Fabrice Bauer ${ }^{15}$, David Montani ${ }^{1,2,3}$, Marc Humbert ${ }^{1,2,3}$ and \\ Gérald Simonneau ${ }^{1,2,3}$
}

\begin{abstract}
Affiliations: ${ }^{1}$ Univ. Paris-Sud, Faculté de Médecine, Université Paris-Saclay, Le Kremlin-Bicêtre, France. ${ }^{2}$ AP-HP, Service de Pneumologie, Hôpital Bicêtre, Le Kremlin-Bicêtre, France. ${ }^{3}$ INSERM UMR_S 999, Hôpital Marie-Lannelongue, Le Plessis-Robinson, France. ${ }^{4}$ Service de Médecine Vasculaire et Thérapeutique, Hôpital Nord, CHU, Saint-Etienne, France. ${ }^{5}$ Univ. Jean-Monnet, Groupe de Recherche sur la Thrombose (EA 3065), INSERM, CIC1408, Saint-Etienne, France. 'Univ. Lyon-1, Hospices Civils de Lyon, Centre de Référence des maladies pulmonaires rares, Centre de Compétences de l'Hypertension Pulmonaire, Hôpital Louis Pradel, Lyon, France. ${ }^{7}$ Hôpital Cardiologique de Lille, Centre de compétences de l'Hypertension Pulmonaire, Lille, France. ${ }^{8} \mathrm{CHU}$ Nancy, Pôle des spécialités médicales, Département de Pneumologie, Vandoeuvre-lès-Nancy, France. ${ }^{9}$ Université de Lorraine, INGRES, EA 7298, Vandoeuvre-lès-Nancy, France. ${ }^{10} \mathrm{CHU}$ Rennes, Service de Cardiologie et Maladies Vasculaires, INSERM U1099, Rennes, France. ${ }^{11}$ Hôpital Côte de Nacre, Centre de compétences Basse Normandie de l'Hypertension Pulmonaire, Université de Caen-Basse Normandie, Caen, France. ${ }^{12}$ Centre Hospitalier Universitaire, Service de cardiologie, Grenoble, France. ${ }^{13} \mathrm{CHU}$ ClermontFerrand, Hôpital Gabriel Montpied, Service de cardiologie et maladies vasculaires, Clermont-Ferrand, France. ${ }_{14}$ Université de Montpellier; INSERM U1046, UMR 9214, Hôpital Arnaud de Villeneuve, Service de pneumologie, Montpellier, France. ${ }^{15}$ Cardiology Dept, Rouen University Health Centre, Rouen, France.
\end{abstract}

Correspondence: Olivier Sitbon, Service de Pneumologie et Soins Intensifs, CHU de Bicêtre, 78 rue du General Leclerc, F-94275 Le Kremlin-Bicêtre, France. E-mail: olivier.sitbondaphp.fr

ABSTRACT Treatment for pulmonary arterial hypertension (PAH) has been underpinned by single-agent therapy to which concomitant drugs are added sequentially when pre-defined treatment goals are not met.

This retrospective analysis of real-world clinical data in 97 patients with newly diagnosed PAH ( $86 \%$ in New York Heart Association functional class III-IV) explored initial dual oral combination treatment with bosentan plus sildenafil $(n=61)$, bosentan plus tadalafil $(n=17)$, ambrisentan plus tadalafil $(n=11)$ or ambrisentan plus sildenafil $(\mathrm{n}=8)$.

All regimens were associated with significant improvements in functional class, exercise capacity, dyspnoea and haemodynamic indices after 4 months of therapy. Over a median follow-up period of 30 months, $75(82 \%)$ patients were still alive, 53 (71\%) of whom received only dual oral combination therapy. Overall survival rates were $97 \%, 94 \%$ and $83 \%$ at 1,2 and 3 years, respectively, and $96 \%, 94 \%$ and $84 \%$, respectively, for the patients with idiopathic $\mathrm{PAH}$, heritable $\mathrm{PAH}$ and anorexigen-induced $\mathrm{PAH}$. Expected survival rates calculated from the French equation for the latter were $86 \%, 75 \%$ and $66 \%$ at 1,2 and 3 years, respectively.

Initial combination of oral PAH-targeted medications may offer clinical benefits, especially in PAH patients with severe haemodynamic impairment.

@ERSpublications

Initial dual combination therapy may offer potential benefits in PAH patients with severe haemodynamic impairment http://ow.ly/YNxgk

Received: Dec 042015 | Accepted after revision: Feb 222016 | First published online: March 172016

Support statement: Medical writing assistance was provided by Moira Anderson and Julia Heagerty (Elements Communications Ltd, Westerham, UK) and was funded by Actelion Pharmaceuticals Ltd. Funding information for this article has been deposited with FundRef.

Conflict of interest: Disclosures can be found alongside the online version of this article at erj.ersjournals.com

Copyright @ERS 2016 


\section{Introduction}

Pulmonary arterial hypertension (PAH) is a rare, progressive and life-limiting disease [1] but one for which treatment options are increasing $[2,3]$. Until 2001, epoprostenol was the only drug available to treat $\mathrm{PAH}$, and then primarily as a bridge to transplantation [4]. Since then, the development of new $\mathrm{PAH}$-specific medications has grown apace. Today, there are nine medications approved in Europe for treatment of PAH, six of which are oral, with others in development. With the advent of new medications, evidence-based guidelines have evolved to reflect the rapidly changing treatment environment $[2,3]$. Observations that patients with an inadequate clinical response to initial single-agent therapy had a much worse prognosis soon led to recommendations to add a second and potentially a third drug to the $\mathrm{PAH}$-specific regimen to improve clinical and functional parameters and long-term outcomes $[5,6]$.

Endothelial dysfunction in the pulmonary bed is believed to act as a major trigger for PAH. The endothelin, nitric oxide and prostacyclin pathways have been implicated in its pathogenesis, with elevated levels of plasma endothelin and lower levels of nitric oxide and prostacyclin metabolites evident in patients with PAH $[7,8]$. Because of these observations and the potential interaction between these three pathways, the combined use of drugs that target the different pathways is an attractive therapeutic option that could increase the effect of targeting one pathway alone and, as a result, may improve treatment outcomes. Studies on the use of sequential combination therapy in patients failing single-agent therapy have yielded mixed results [9]. A meta-analysis of six randomised controlled trials on combination therapy, which included 858 patients with $\mathrm{PAH}$, showed that in comparison with the control group, combination therapy significantly improved exercise capacity and haemodynamics as well as reducing the risk of clinical worsening. However, there was no significant reduction in all-cause mortality [10].

Real-life data from registries or single-centre patient databases show that combination therapy has been widely adopted in clinical practice, with rates ranging from $46-75 \%$ in patients with idiopathic PAH (IPAH) $[11,12]$ to $29-50 \%$ in patients with PAH associated with connective tissue disease (PAH-CTD) $[13,14]$ and $17-32 \%$ in patients with $\mathrm{PAH}$ associated with congenital heart disease [15].

Most data on the use of combination therapy are based on sequential addition to ongoing single-agent therapy, rather than initial combination therapy, although there is a strong case for initiating combination therapy to increase efficacy and optimise outcome [16]. In fact, this practice is gaining in popularity, especially in France, with more patients than ever being treated with first-line dual (and in some cases triple) therapy. Data now emerging from the Ambrisentan and Tadalafil in Patients with Pulmonary Arterial Hypertension (AMBITION) trial, the first randomised controlled trial of initial combination dual therapy, show a highly significant reduction in the risk of clinical failure for patients on first-line dual therapy, as compared with patients on monotherapy [17]. The study did not, however, include haemodynamic indices as outcome measures.

In this retrospective analysis of real-world clinical data, we explored the effect of different combinations of first-line PAH-specific dual oral therapy on haemodynamic parameters and clinical and functional outcomes in newly diagnosed patients with PAH.

\section{Methods}

This study complied with the Declaration of Helsinki. Although French law does not require ethics committee approval or informed consent for retrospective data collection, the data collected were anonymised and complied according to the requirements of the Commission Nationale Informatique et Liberté (CNIL), the organisation dedicated to privacy, information technology and civil rights in France. CNIL approved the methods used to collect and analyse data on May 24, 2003 (approval number 842063).

\section{Study design}

This was a retrospective analysis of data from consecutive newly diagnosed PAH patients (incident cases) admitted to the reference centre (University Paris-Sud, Le Kremlin-Bicêtre, France) and 15 expert centres from the French Pulmonary Hypertension Network who were initiated on first-line dual oral combination therapy consisting of an endothelin receptor antagonist (ERA) (bosentan or ambrisentan) and a phosphodiesterase type 5 (PDE5) inhibitor (sildenafil or tadalafil). Patients meeting inclusion criteria were enrolled between January 2007 and December 2013. The observational period ended 12 months after enrolment of the final patient.

\section{Patients}

Eligible patients included adults aged over 18 years with confirmed PAH of any aetiology and in New York Heart Association functional class (NYHA FC) II-IV. Women who were pregnant and those of childbearing age not using an adequate method of birth control were excluded. Other exclusion criteria included patients with non-group 1 pulmonary hypertension and pulmonary veno-occlusive 
disease as well as unstable patients in need of parenteral prostacyclin and those for whom ERAs were contraindicated (i.e. liver enzyme concentrations more than three times the upper limit of normal (ULN)).

\section{Treatment regimens}

Choice of PAH-specific dual therapy was at the discretion of the treating physician and included one of four oral regimens: bosentan plus sildenafil, bosentan plus tadalafil, ambrisentan plus sildenafil and ambrisentan plus tadalafil. Within these regimens, therapies were given concomitantly, starting with two drugs on day 1. Dosing was: bosentan $62.5 \mathrm{mg}$ twice daily, increasing to $125 \mathrm{mg}$ twice daily after 4 weeks; ambrisentan $5 \mathrm{mg}$ once daily, increasing to $10 \mathrm{mg}$ once daily if needed (in cases of insufficient clinical and/or haemodynamic response at the first follow-up visit) and there were no tolerability issues; sildenafil $20 \mathrm{mg}$ three times daily, increasing to $40 \mathrm{mg}$ three times daily if needed (in cases of insufficient clinical and/or haemodynamic response at the first follow-up visit); or tadalafil $20 \mathrm{mg}$ once daily with up-titration to $40 \mathrm{mg}$ once daily after 3-7 days, according to tolerability. In addition to PAH-specific therapy, if needed, patients also received standard supportive therapy that included oral anticoagulants, diuretics and oxygen. Patients were also eligible to receive add-on therapy with prostanoids if their condition deteriorated or they had an inadequate response while on dual therapy. At the discretion of the treating physician, patients could receive intravenous, subcutaneous or inhaled prostanoids.

\section{Study assessments}

Patients were screened before initiation of therapy (baseline), 4 months after initiation of combination therapy (first follow-up visit) and once each year until the end of the study. All patients underwent acute pulmonary vasoreactivity testing with inhaled nitric oxide at the time of their first haemodynamic evaluation and all were found to be nonvasoreactive.

The last follow-up visit was defined as the time-point where a complete evaluation (including right heart catheterisation (RHC)) had been conducted. This was performed while patients were on dual oral combination therapy and before recourse to add-on prostanoid therapy where that was deemed necessary. Assessments included NYHA FC, 6-min walk distance (6MWD) and pulmonary haemodynamics (assessed by RHC). If patients were unable to perform the 6-min walk test at baseline or at any of the follow-up visits, a $6 \mathrm{MWD}$ of $0 \mathrm{~m}$ was recorded. Adverse events were recorded throughout the study period.

\section{Statistical analysis}

Data were stored in a personal computer-based data spreadsheet. Analysis was performed using the StatEL statistical package (Ad Science, Paris, France) in Excel 2007 (Microsoft Corp., Redmond, WA, USA). All data are presented as mean \pm SD. Comparison of $6 \mathrm{MWD}$ and haemodynamic variables obtained at baseline, first follow-up and last follow-up visits was made using one-way ANOVA for paired groups for normally distributed variables and the nonparametric Friedman test for the non-normally distributed variables. Post hoc comparisons were made using the Newman and Keuls test or nonparametric Wilcoxon test, as appropriate. The Chi-squared test for independence was used to compare differences between NYHA FC assessed at the baseline, first follow-up and last follow-up visits. The comparisons of combined bosentan and PDE5 inhibitor versus ambrisentan and PDE5 inhibitor, and combined sildenafil and ERA versus tadalafil and ERA were analysed by ANOVA adjusted for baseline values. A p-value $<0.05$ was considered statistically significant.

Analysis of overall survival was performed using an intention-to-treat approach and was thus independent of the treatment regimen patients were receiving at that time (i.e. patients could be receiving add-on therapy). For the survival analysis, the date of diagnostic RHC $(<1$ week before commencement of treatment) was used as the starting point to determine length of survival. The cut-off date was December 31,2014 , or the date of death or transplantation if that was earlier. The Kaplan-Meier method was used to estimate overall survival at each interval. Patients who underwent lung transplantation were considered censored at the date of transplantation. Patients who were lost to follow-up were considered censored at the last available visit. Expected survival was calculated using a survival prediction equation (commonly referred to as the French equation) using three variables: sex, 6MWD at diagnosis and cardiac output at diagnosis, as previously described [18, 19]. Comparison of actual and expected survival was only computed for patients with IPAH, heritable PAH and anorexigen-induced PAH, as the equation is not valid for other forms of PAH.

\section{Results}

Patient demographics and characteristics at baseline

A total of 97 patients met the eligibility criteria for inclusion in this retrospective analysis, the details of which are summarised in table 1 . Consistent with the higher prevalence rates of PAH among women, almost 
TABLE 1 Demographics and baseline characteristics of the 97 patients with newly diagnosed pulmonary arterial hypertension (PAH) initiated with endothelin receptor antagonist and phosphodiesterase type 5 inhibitor dual combination therapy

$\begin{array}{lc}\text { Male/female } & 34(35) / 63(65) \\ \text { Age years } & 54.1 \pm 16.5 \\ \text { Body mass index } \mathbf{~ k g} \cdot \mathbf{m}^{-2} & 27.5 \pm 6.6 \\ >30 \mathrm{~kg} \cdot \mathrm{m}^{-2} & 28(29) \\ \text { PAH diagnosis, } \mathbf{n ~ ( \% )} & 52(54) \\ \text { Idiopathic PAH } & 15(15) \\ \text { Heritable PAH } & 7(7) \\ \text { Anorexigen-induced PAH } & 12(12) \\ \text { PAH-CTD } & 9(9) \\ \text { Portopulmonary hypertension } & 1(1) \\ \text { Repaired PAH-CHD } & 1(1) \\ \text { PAH-HIV } & 15(15) \\ \text { NYHA FC } & 70(72) \\ \text { II } & 12(12) \\ \text { III } & 49(51) \\ \text { IV } & 324 \pm 132 \\ \text { Clinical signs of right heart failure } & 4.3 \pm 2.0 \\ \text { 6-min walk distance m } & 372(115-710) \\ \text { Borg dyspnoea index } & \end{array}$

Data are presented as $\mathrm{n}(\%)$ or mean \pm SD, unless otherwise stated. CTD: connective tissue disease; CHD: congenital heart disease; NYHA FC: New York Heart Association functional class; BNP: brain natriuretic peptide; IQR: interquartile range. ${ }^{\#}: \mathrm{n}=42$.

two-thirds of the patients were female. A majority of patients had IPAH (54\%), followed by heritable PAH (15\%). A majority (86\%) were in NYHA FC III-IV and had severe haemodynamic impairment at referral.

\section{Treatment}

In total, 61 patients received bosentan in combination with sildenafil, 17 patients received bosentan plus tadalafil, 11 patients received ambrisentan plus tadalafil and eight patients received ambrisentan plus sildenafil. A majority of patients (72\%) also received supportive therapy consisting of oral anticoagulants, diuretics and oxygen. At the first follow-up visit, all patients on bosentan were receiving $125 \mathrm{mg}$ twice daily. Six patients initiated on ambrisentan $5 \mathrm{mg}$ once daily were up-titrated to $10 \mathrm{mg}$ once daily due to insufficient clinical and/or haemodynamic responses. All patients but one initiated on tadalafil (who remained on $20 \mathrm{mg}$ ) were up-titrated to $40 \mathrm{mg}$ once daily within 1 week. Sildenafil was initiated at $20 \mathrm{mg}$ three times daily in all patients. Only three patients were up-titrated to $40 \mathrm{mg}$ three times daily due to insufficient clinical and/or haemodynamic responses at the first follow-up visit.

\section{Treatment response at first follow-up visit}

Of the 97 patients who received PAH-specific dual therapy, all had a first follow-up visit at 4 months (median 4.1 months, interquartile range (IQR) 3.5-4.9 months). Combination therapy (pooled analysis) was associated with significant improvements in functional class, exercise capacity and dyspnoea. Significantly fewer patients had evidence of right heart failure (suggesting a reduction in clinical worsening), while levels of brain natriuretic peptide (BNP) were significantly reduced (table 2). These improvements were accompanied by significant improvements in 6MWD and haemodynamic parameters, including reductions from baseline in pulmonary vascular resistance (PVR). Whereas no difference was found when PDE5 inhibitors were combined with either bosentan or ambrisentan, the combination of ERAs with tadalafil was associated with a significantly greater reduction in PVR compared with sildenafil and an ERA (table 3).

\section{Treatment response at last follow-up visit}

Of the 97 patients assessed at 4 months, 20 had no further haemodynamic assessment: six (6\%) patients were lost to long-term follow-up, eight had died and six were still alive, with a follow-up ranging from 12 to 19 months. Therefore, a complete reassessment of dual oral combination therapy was available in 77 patients (median follow-up 24.6 months, IQR 15.8-37.4 months) (table 4). Despite a slight increase in mean pulmonary arterial pressure (mPAP) and PVR, improvements observed at first follow-up visit were sustained in these patients. 
TABLE 2 Effect of initial pulmonary arterial hypertension-specific dual oral combination therapy on New York Heart Association functional class (NYHA FC), symptoms, exercise capacity, brain natriuretic peptide (BNP) and haemodynamics at first follow-up visit ( $\mathrm{n}=97$ )

\begin{tabular}{|c|c|c|c|}
\hline & Baseline & First follow-up visit ${ }^{\#}$ & $p$-value \\
\hline NYHA FC I/II/III/IV n & 0/15/70/12 & $4 / 57 / 31 / 5$ & $<0.001$ \\
\hline Clinical signs of RHF n (\%) & $49(51)$ & $25(26)$ & $<0.001$ \\
\hline 6-min walk distance m & $324 \pm 132$ & $395 \pm 114$ & $<0.00001$ \\
\hline Borg dyspnoea index & $4.3 \pm 2.0$ & $3.1 \pm 1.9$ & $<0.00001$ \\
\hline BNP $P^{\pi} \mathrm{ng} \cdot \mathrm{L}^{-1}$ median (IQR) & $372(115-710)$ & $62(34-274)$ & $<0.00001$ \\
\hline \multicolumn{4}{|l|}{ Haemodynamics } \\
\hline $\mathrm{RAP} \mathrm{mmHg}$ & $9.5 \pm 5.7$ & $6.7 \pm 4.5$ & $<0.00001$ \\
\hline mPAP $\mathrm{mmHg}$ & $53.9 \pm 10.4$ & $45.1 \pm 10.9$ & $<0.00001$ \\
\hline PAWP mmHg & $8.8 \pm 3.5$ & $8.7 \pm 3.3$ & 0.82 \\
\hline Cardiac output L. $\min ^{-1}$ & $3.94 \pm 1.17$ & $5.65 \pm 1.62$ & $<0.00001$ \\
\hline Cardiac index $\mathrm{L} \cdot \mathrm{min}^{-1} \cdot \mathrm{m}^{-2}$ & $2.14 \pm 0.51$ & $3.13 \pm 0.79$ & $<0.00001$ \\
\hline PVR dyn $\cdot \mathrm{s} \cdot \mathrm{cm}^{-5}$ & $1021 \pm 357$ & $565 \pm 252$ & $<0.00001$ \\
\hline Mean $\mathrm{BP} \mathrm{mmHg}$ & $97 \pm 18$ & $87 \pm 13$ & $<0.00001$ \\
\hline Heart rate beats per min & $85 \pm 15$ & $81 \pm 12$ & $<0.011$ \\
\hline $\mathrm{SvO}_{2} \%$ & $59 \pm 8$ & $67 \pm 8$ & $<0.00001$ \\
\hline
\end{tabular}

Data are presented as mean \pm SD unless otherwise stated. RHF: right heart failure; IQR: interquartile range; RAP: right atrial pressure; mPAP: mean pulmonary artery pressure; PAWP: pulmonary artery wedge pressure; PVR: pulmonary vascular resistance; BP: blood pressure; $\mathrm{SvO}_{2}$ : mixed venous oxygen saturation. \#: median follow-up time 4.1 months (IQR 3.5-4.9 months); ${ }^{\text {१: }} \mathrm{n}=42$.

\section{Long-term follow-up}

Over a median (IQR) observational follow-up period of 30 (20-43) months, 75 (82\%) patients were still alive. Among survivors, 53 received only dual oral combination therapy, while 22 had their treatment escalated with additional prostanoid therapy (i.v. epoprostenol $(n=8)$, subcutaneous treprostinil $(n=5)$, inhaled iloprost $(n=5)$ or selexipag as part of the GRIPHON (Prostaglandin $\mathrm{I}_{2}$ Receptor Agonist in Pulmonary Arterial Hypertension) randomised controlled trial $(n=4)$ [20]). Of the 16 patients who died during long-term follow-up, 11 had IPAH, three had PAH associated with systemic sclerosis and two had portopulmonary hypertension. At the time of death, 11 patients received only dual oral combination therapy while five received additional prostanoid therapy. Causes of death were as follows: right heart failure $(n=8)$, sepsis $(n=2)$, sudden death $(n=1)$, atrioventricular block $(n=1)$, cancer $(n=2)$ and unknown $(n=2)$. Amongst the eight patients who died from right heart failure, five had received additional prostanoid therapy. Prostacyclin was not prescribed because of advanced age in one patient and blindness in another. No patient underwent lung transplantation. Overall survival rates were $97 \%, 94 \%$ and $83 \%$ at 1,2 and 3 years, respectively (figure 1). Separate calculations for the patients with IPAH, heritable PAH and anorexigen-induced PAH were 96\%, 94\%

TABLE 3 The effect of initial pulmonary arterial hypertension-specific dual oral combination therapy regimens on haemodynamic parameters at first follow-up visit

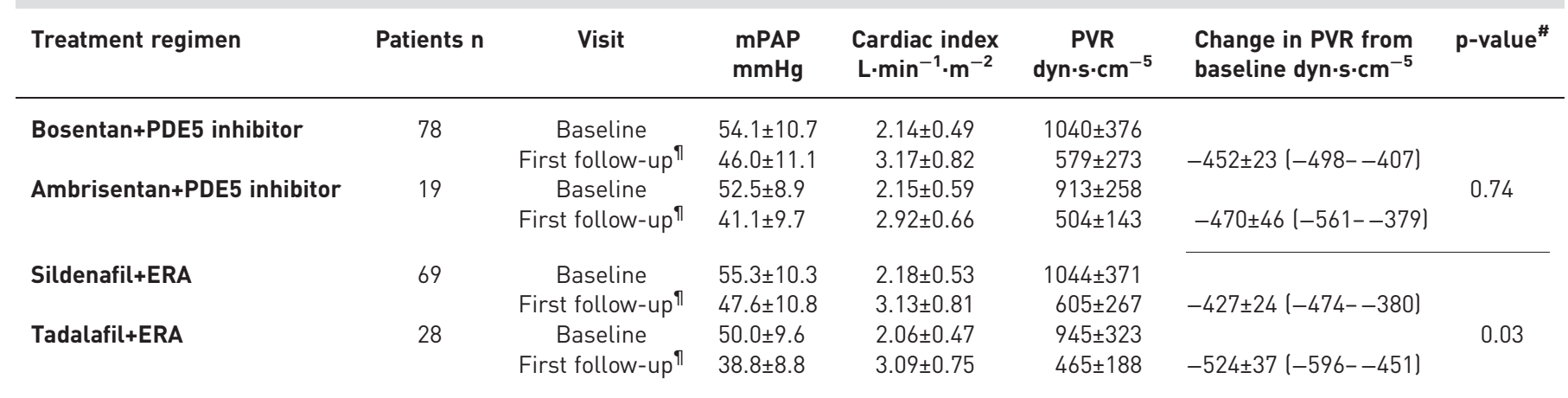

Data are presented as mean \pm SD or mean \pm SD $(95 \% \mathrm{Cl})$ unless otherwise stated. mPAP: mean pulmonary artery pressure; PVR: pulmonary vascular resistance; PDE5: phosphodiesterase type 5; ERA: endothelin receptor antagonist. \# : comparisons of combined bosentan and PDE5 inhibitor versus ambrisentan and PDE5 inhibitor, and combined sildenafil and ERA versus tadalafil and ERA (ANOVA adjusted for baseline values); ף: median follow-up time 4.1 months (interquartile range 3.5-4.9 months). 
TABLE 4 Effect of initial pulmonary arterial hypertension-specific dual oral combination therapy on New York Heart Association function class (NYHA FC), symptoms, exercise capacity and haemodynamics at first and last follow-up visits $(n=77)$

\begin{tabular}{|c|c|c|c|c|}
\hline & Baseline & First follow-up visit ${ }^{\#}$ & Last follow-up visit ${ }^{\pi}$ & p-value \\
\hline NYHA FC I/II/III/IV n & $0 / 11 / 57 / 9$ & $4 / 49 / 22 / 2$ & $10 / 37 / 26 / 4$ & $<0.00001$ \\
\hline Clinical signs of RHF $n(\%)$ & $35(45)$ & $15(19)$ & $16(21)$ & $<0.0004$ \\
\hline 6-min walk distance $\mathrm{m}$ & $354 \pm 127$ & $427 \pm 107 * *$ & $438 \pm 117^{* *}$ & $<0.00001$ \\
\hline Borg dyspnoea index & $4.4 \pm 2.1$ & $3.0 \pm 2.0 * *$ & $3.2 \pm 1.9 * *$ & $<0.0003$ \\
\hline \multicolumn{5}{|l|}{ Haemodynamics } \\
\hline RAP $\mathrm{mmHg}$ & $9.1 \pm 6.0$ & $6.1 \pm 3.9 * *$ & $7.7 \pm 4.5$ & $<0.00008$ \\
\hline mPAP $\mathrm{mmHg}$ & $54.2 \pm 11.1$ & $44.6 \pm 11.3^{* *}$ & $47.4 \pm 13.3^{* * * *}$ & $<0.00001$ \\
\hline PAWP mmHg & $8.9 \pm 3.6$ & $8.8 \pm 3.5$ & $9.2 \pm 3.8$ & 0.67 \\
\hline Cardiac output L. $\min ^{-1}$ & $3.93 \pm 1.09$ & $5.74 \pm 1.75^{* *}$ & $5.71 \pm 2.00 * *$ & $<0.00001$ \\
\hline Cardiac index $L \cdot \min ^{-1} \cdot \mathrm{m}^{-2}$ & $2.14 \pm 0.49$ & $3.14 \pm 0.85^{* *}$ & $3.07 \pm 0.91 * *$ & $<0.00001$ \\
\hline PVR dyn $\cdot s \cdot \mathrm{cm}^{-5}$ & $978 \pm 304$ & $536 \pm 215^{* *}$ & $618 \pm 332^{*, * *}$ & $<0.00001$ \\
\hline Mean BP mmHg & $94 \pm 17$ & $87 \pm 14^{* *}$ & $89 \pm 14^{* *}$ & $<0.0003$ \\
\hline Heart rate beats per min & $86 \pm 15$ & $79 \pm 12^{* *}$ & $79 \pm 12^{* *}$ & $<0.0006$ \\
\hline $\mathrm{SvO}_{2} \%$ & $61 \pm 9$ & $70 \pm 5^{* *}$ & $69 \pm 7^{* *}$ & $<0.00001$ \\
\hline
\end{tabular}

Data are presented as mean \pm SD unless otherwise stated. RHF: right heart failure; RAP: right atrial pressure; mPAP: mean pulmonary artery pressure; PAWP: pulmonary artery wedge pressure; PVR: pulmonary vascular resistance; $\mathrm{BP}$ : blood pressure; $\mathrm{SvO}_{2}$ : mixed venous oxygen saturation. \#: median follow-up time 4.1 months (interquartile range 3.5-4.9 months); ๆ: median follow-up time 24.6 months (interquartile range 15.8-37.4 months). *: $p<0.05$, comparison with first follow-up visit value; ${ }^{* *}: p<0.01$, comparison with baseline value.

and $84 \%$ at 1,2 and 3 years, respectively. Expected survival rates calculated from the French equation for these patients were $86 \%, 75 \%$ and $66 \%$ at 1,2 and 3 years, respectively (figure 2 ).

\section{Safety and tolerability}

PAH-specific dual therapy with ERAs in combination with PDE5 inhibitors was generally well tolerated with adverse events consistent with the drugs' known side-effect profiles. During follow-up, one patient stopped sildenafil due to ocular problems (blurred vision). She remained stable in NYHA FC II on bosentan monotherapy. Nine patients initially receiving sildenafil were transitioned to tadalafil, mainly to facilitate the treatment regimen (once a day versus three times a day). Two patients were switched from bosentan to ambrisentan due to liver enzyme elevation more than five times the ULN. Two other patients were switched

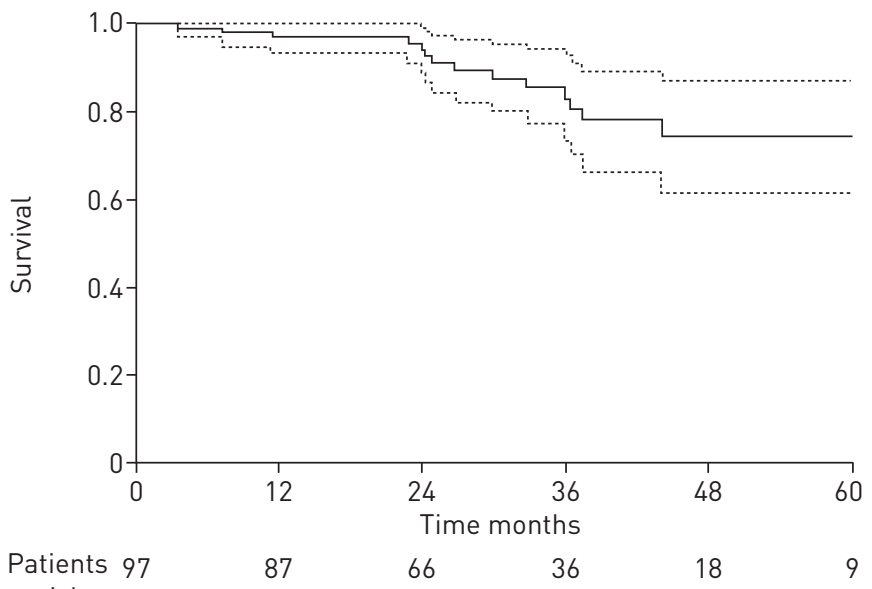

at risk $n$

FIGURE 1 Overall survival rates in all patients with newly diagnosed pulmonary arterial hypertension following initial dual oral combination therapy with endothelin receptor antagonist and phosphodiesterase type 5 inhibitor (n=97). The solid line denotes the actual survival and dashed lines the 95\% confidence interval. Survival was $97 \%(95 \% \mathrm{Cl} 93-100 \%), 94 \%(95 \% \mathrm{Cl} 89-99 \%)$ and $83 \%(95 \% \mathrm{Cl} 73-93 \%)$ at 1,2 and 3 years, respectively. 


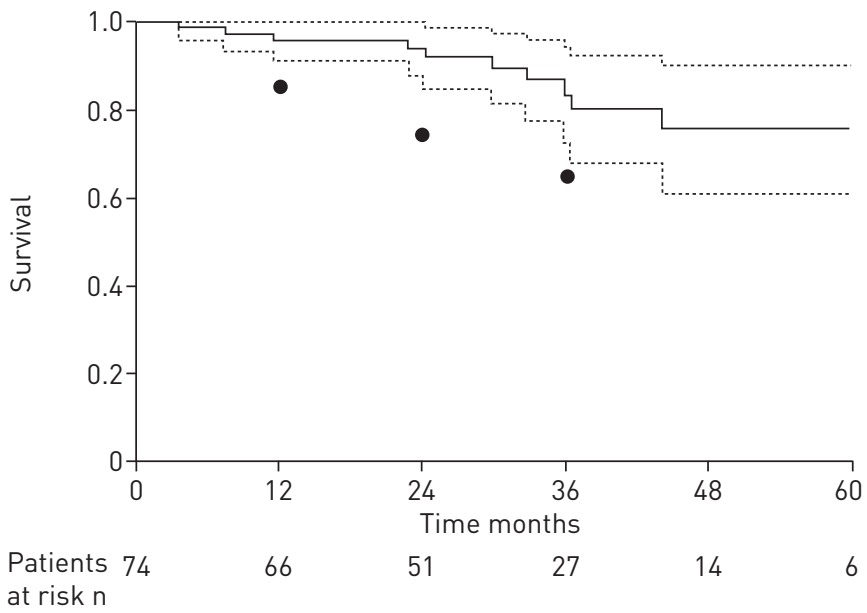

FIGURE 2 Actual and expected survival rates of patients with idiopathic pulmonary arterial hypertension (PAH), heritable PAH and anorexigen-induced PAH following initial dual oral combination therapy with endothelin receptor antagonist and phosphodiesterase type 5 inhibitor $(n=74)$. The solid line denotes the actual survival and dashed lines the $95 \%$ confidence interval. Circles denote the expected survival rates at 1 , 2 and 3 years according to a survival prediction equation (commonly referred to as the French equation), as previously described $[18,19]$. Actual survival was $96 \%(95 \% \mathrm{Cl} 91-100 \%), 94 \%(95 \% \mathrm{Cl} 88-99 \%)$ and $84 \%$ $(95 \% \mathrm{Cl} 72-95 \%)$ at 1,2 and 3 years, respectively. For comparison, predicted survival was $86 \%(95 \% \mathrm{Cl} 83$ $-88 \%), 75 \%(95 \% \mathrm{Cl} 71-79 \%)$ and $66 \%(95 \% \mathrm{Cl} 62-71 \%)$ at 1,2 and 3 years, respectively.

from ambrisentan to bosentan due to leg oedema. All switches between ERAs led to resolution of the adverse event. In all cases of medication change, patients remained stable with no signs of clinical deterioration.

\section{Discussion}

Data from retrospective analyses have shown that long-term outcomes of patients with PAH are strongly correlated with NYHA FC and exercise capacity, in which right ventricular dysfunction is believed to account for the effects of functional class and exercise capacity on mortality [21, 22]. An important goal of therapy is therefore to improve haemodynamic indices of right ventricular function leading to commensurate improvement in NYHA FC and exercise capacity; the latter are important end-points as they provide an indication of patient well-being. As a guideline, several indices have been suggested as appropriate treatment targets and have evidence of survival benefit [21-25]. These include an NYHA FC of I or II, a $6 \mathrm{MWD}$ of $\geqslant 380-440 \mathrm{~m}$, a cardiac index of more than $2.5-3.0 \mathrm{~L} \cdot \mathrm{min}^{-1} \cdot \mathrm{m}^{-2}$, a right atrial pressure (RAP) of $<8 \mathrm{mmHg}$ and levels of BNP in the normal range $\left(0-99 \mathrm{ng} \cdot \mathrm{L}^{-1}\right)$.

Results of this retrospective analysis of 97 patients with newly diagnosed PAH of varying aetiology showed that first-line oral combination therapy of an ERA plus a PDE5 inhibitor is associated with significant improvements in all of the aforementioned indices. At first reassessment, mean 6MWD exceeded $380 \mathrm{~m}$, RAP declined to $6.7 \mathrm{mmHg}$ and cardiac index increased to $3.13 \mathrm{~L} \cdot \mathrm{min}^{-1} \cdot \mathrm{m}^{-2}$ (reflecting normalisation of right ventricular function at rest), while BNP levels decreased from a median value of $372 \mathrm{ng} \cdot \mathrm{L}^{-1}$ before treatment to $62 \mathrm{ng} \cdot \mathrm{L}^{-1}$ at 4 months. Reductions in PVR were significant with all treatment regimens and exceeded $40 \%$, consistent with the findings from other comparable studies [2]. Available evidence suggests that PVR typically falls by about $30 \%$ on PAH-specific monotherapy, to $40-50 \%$ on dual therapy [26, 27], and by almost $70 \%$ on triple therapy [16]. First-line dual oral combination therapy was also associated with significant reductions in mPAP, which appeared greater than the modest reductions (typically 2 $5 \mathrm{mmHg}$ ) documented for single-agent therapy [28]. However, the maximum reduction of $11 \mathrm{mmHg}$ in mPAP observed after 4 months therapy with tadalafil plus ERA was not as great as the average $>20 \mathrm{mmHg}$ reduction observed with initial triple therapy in a similar patient population [16].

In our study, patients receiving tadalafil appeared to exhibit a greater haemodynamic improvement than those on sildenafil, irrespective of the concomitant ERA. This could be explained by the fact that all patients on sildenafil were initiated on the approved dose of $20 \mathrm{mg}$ three times daily. In the pivotal randomised controlled trial of sildenafil versus placebo (SUPER-1), the change from baseline in PVR was dose dependent, from $-12 \%$ with the lowest dose of $20 \mathrm{mg}$ three times daily to $-28 \%$ with the highest dose of $80 \mathrm{mg}$ three times daily [29]. Interestingly, in the haemodynamic substudy of the randomised controlled trial with tadalafil (PHIRST), the decrease from baseline in PVR was $25-30 \%$ in patients receiving tadalafil $20 \mathrm{mg}$ or $40 \mathrm{mg}$ once daily [30]. This difference in haemodynamic effect may explain our observations with respect to sildenafil and tadalafil treatment regimens. 
At referral, 82 patients were in NYHA FC III and IV. Within 4 months of treatment, 61 (74.4\%) patients were in NYHA FC I or II. These significant improvements occurred irrespective of the dual combination therapy regimen, suggesting a class effect. Improvements in haemodynamic indices and, in turn, NYHA FC are associated with a better prognosis and this is supported by our survival data, which showed that patients on initial dual combination therapy had better survival prospects than would have been expected based on the equation derived from the French Registry for newly diagnosed (incident idiopathic, heritable and anorexigen-induced cases). There are, as yet, only a limited number of studies that have explored the potential benefits of using first-line combination therapy as an alternative to sequential addition. Nonetheless, the present study adds to a growing body of evidence to suggest that initial combination therapy is a viable strategy for patients with $\mathrm{PAH}$ with potential to delay clinical worsening.

In BREATHE-2, a prospective, double-blind, placebo-controlled study that compared initial bosentan plus epoprostenol versus epoprostenol alone in severe $\mathrm{PAH}$, there was a trend (although nonsignificant) towards greater improvement in haemodynamic parameters, exercise capacity and NYHA FC after 16 weeks' combination treatment [31].

Data from the AMBITION trial provide more robust evidence of clinical benefit, albeit without any supporting haemodynamic data. In this large-scale, prospective, double-blind study, treatment-naïve patients with NYHA FC II and III PAH were randomised to either first-line combination therapy (ambrisentan and tadalafil) or first-line monotherapy with ambrisentan or tadalafil. The primary end-point was the time to the first clinical failure event, which included the time from randomisation to the first occurrence of death (all-cause), hospitalisation for worsening $\mathrm{PAH}$, disease progression or unsatisfactory long-term clinical response. Results showed a significant difference between combination therapy and monotherapy (pooled single-therapy arms) for the primary end-point $(\mathrm{p}<0.01)$, driven primarily by a $63 \%$ reduction in hospitalisation for worsening PAH $(\mathrm{p}=0.0002)$. Overall, initial combination therapy reduced the risk of clinical failure by $50 \%(\mathrm{p}=0.0002)$. These improvements were achieved without compromising safety or tolerability [17]. A small, single-centre haemodynamic substudy from the AMBITION trial showed that initial combination therapy with ambrisentan and tadalafil was associated with larger improvements in haemodynamics and exercise capacity compared with monotherapy [32].

These data are supported by results from a number of noncontrolled prospective and retrospective analyses. A retrospective study of 23 subjects initiated on epoprostenol plus bosentan compared with matched historical controls treated with epoprostenol alone showed that dual therapy with bosentan and epoprostenol improved patients' haemodynamics, NYHA FC, exercise capacity and clinical status over 4 months [26]. PVR was significantly improved in patients on dual therapy compared with patients on epoprostenol alone $(-48 \pm 17 \%$ versus $-29 \pm 17 \%$, respectively; $\mathrm{p}=0.0001)$. However, no statistically significant difference was observed between treatment arms with respect to survival or transplant-free survival. Results from a prospective, nonrandomised, observational study of 43 treatment-naïve subjects, in which 26 received dual therapy with epoprostenol plus oral treatment (ERA $(n=16)$, PDE5 ( $n=1)$ or both ERA and PDE5 $(n=9)$ ), showed significantly greater haemodynamic improvement in the combination group compared with epoprostenol alone $(\mathrm{p}=0.03)$ and a trend towards improved exercise capacity [33]. Results from a retrospective analysis of 77 patient records in Sweden between 2000 and 2011 showed that initial combination therapy $(n=13)$ was associated with significantly greater reductions in PVR $(p=0.017)$ and significantly improved cardiac index $(p=0.04)$ [34]. A recent open-label study analysed the efficacy and safety of initial combination therapy with ambrisentan and tadalafil in patients with PAH-CTD and showed marked haemodynamic improvements with this strategy [27].

Although PAH remains an incurable disease, survival rates continue to improve. In 1986, median survival for IPAH was only 2.8 years [35], whereas it now exceeds 7 years in the REVEAL (Registry to Evaluate Early And Long-term PAH Disease Management) in the USA [36]. As we observed, patients initiated on dual combination therapy have better survival prospects than would have been expected based on the equation derived from the French Registry for newly diagnosed (incident cases). Nonetheless, our survival rate of $83-84 \%$ at 3 years is below the $100 \%$ overall and $94 \%$ transplant-free survival rates observed for a similar cohort of patients treated with initial triple therapy including i.v. prostacyclin [16]. This raises the interesting question of whether patients with $\mathrm{PAH}$, and especially those with severe haemodynamic impairment (i.e. RAP $>14 \mathrm{mmHg}$, cardiac index $<2.0 \mathrm{~L} \cdot \mathrm{min}^{-1} \cdot \mathrm{m}^{-2}$ or mixed venous oxygen saturation $<60 \%$ ), should be started routinely on three therapies (including i.v. prostacyclin) rather than two oral PAH-specific therapies, as recommended in the 2015 European Society of Cardiology/European Respiratory Society pulmonary hypertension guidelines for patients considered to be at high risk of death (i.e. estimated 1-year mortality $>10 \%)[2,3]$.

Our study has limitations requiring cautious interpretation of our findings. The study was retrospective in design and was conducted in a heterogeneous population of group 1 pulmonary hypertension patients, including those with IPAH, heritable PAH, anorexigen-induced PAH, PAH associated with CTD, congenital 
heart disease or HIV, and portopulmonary hypertension. Factors associated with these different aetiologies could have had an impact on our observations. In addition, this study reflects physician treatment choices across France, which has contributed to an imbalance with respect to treatment regimen, with a large proportion of patients receiving initial dual therapy with bosentan and sildenafil. This imbalance may also be due to the timescale of availability of the different drugs and the relative experience with their use.

In patients with $\mathrm{PAH}$, the standard of care has been single-agent therapy with concomitant drugs added sequentially when patients fail to meet pre-defined treatment goals. Results of this retrospective analysis of initial dual oral combination therapy suggest that simultaneous initiation of combined ERA and PDE5 inhibitor therapy may offer benefits, especially in patients with severe haemodynamic impairment for whom prognosis with standard regimens is still poor. All initial dual drug regimens were effective. The combination of tadalafil with bosentan or ambrisentan seems to be associated with a greater haemodynamic improvement than a sildenafil combination regimen when utilised at the recommended dose.

\section{Acknowledgements}

This study was supported in part by the Département Hospitalo-Universitaire Thorax Innovation and the Laboratoire d’Excellence en Recherche sur le Médicament et l'Innovation Thérapeutique.

We thank Laurence Rottat (AP-HP, Paris, France) for her hard work in managing the French Registry and Pierre Clerson (Soladis, Lyon, France) for helping us with the statistics.

\section{References}

$1 \quad$ Humbert M. The burden of pulmonary hypertension. Eur Respir J 2007; 30: 1-2.

2 Galiè N, Humbert M, Vachiery JL, et al. 2015 ESC/ERS Guidelines for the diagnosis and treatment of pulmonary hypertension: The Joint Task Force for the Diagnosis and Treatment of Pulmonary Hypertension of the European Society of Cardiology (ESC) and the European Respiratory Society (ERS) Endorsed by: Association for European Paediatric and Congenital Cardiology (AEPC), International Society for Heart and Lung Transplantation (ISHLT). Eur Heart J 2016; 37: 67-119.

3 Galiè N, Humbert M, Vachiery JL, et al. 2015 ESC/ERS Guidelines for the diagnosis and treatment of pulmonary hypertension. Eur Respir J 2015; 46: 903-975.

4 Rich S. Medical treatment of primary pulmonary hypertension: a bridge to transplantation? Am J Cardiol 1995; 75: 63A-66A.

5 Galiè N, Corris PA, Frost A, et al. Updated treatment algorithm of pulmonary arterial hypertension. J Am Coll Cardiol 2013; 62: D60-D72.

6 Dardi F, Manes A, Palazzini M, et al. Combining bosentan and sildenafil in pulmonary arterial hypertension patients failing monotherapy: real-world insights. Eur Respir J 2015; 46: 414-421.

7 Humbert M, Sitbon O, Simonneau G. Treatment of pulmonary arterial hypertension. N Engl J Med 2004; 351: $1425-1436$.

8 Humbert M, Lau EM, Montani D, et al. Advances in therapeutic interventions for patients with pulmonary arterial hypertension. Circulation 2014; 130: 2189-2208.

9 Ghofrani HA, Humbert M. The role of combination therapy in managing pulmonary arterial hypertension. Eur Respir Rev 2014; 23: 469-475.

10 Galiè N, Palazzini M, Manes A. Pulmonary arterial hypertension: from the kingdom of the near-dead to multiple clinical trial meta-analyses. Eur Heart J 2010; 31: 2080-2086.

11 Ling Y, Johnson MK, Kiely DG, et al. Changing demographics, epidemiology, and survival of incident pulmonary arterial hypertension: results from the pulmonary hypertension registry of the United Kingdom and Ireland. Am J Respir Crit Care Med 2012; 186: 790-796.

12 Ogawa A, Ejiri K, Matsubara H. Long-term patient survival with idiopathic/heritable pulmonary arterial hypertension treated at a single center in Japan. Life Sci 2014; 118: 414-419.

13 Ngian G-S, Stevens W, Prior D, et al. Predictors of mortality in connective tissue disease-associated pulmonary arterial hypertension: a cohort study. Arthritis Res Ther 2012; 14: R213.

14 Campo A, Mathai SC, Le Pavec J, et al. Hemodynamic predictors of survival in scleroderma-related pulmonary arterial hypertension. Am J Respir Crit Care Med 2010; 182: 252-260.

15 Manes A, Palazzini M, Leci E, et al. Current era survival of patients with pulmonary arterial hypertension associated with congenital heart disease: a comparison between clinical subgroups. Eur Heart J 2014; 35: 716-724.

16 Sitbon O, Jaïs X, Savale L, et al. Upfront triple combination therapy in pulmonary arterial hypertension: a pilot study. Eur Respir J 2014; 43: 1691-1697.

17 Galiè N, Barberà JA, Frost AE, et al. Initial use of ambrisentan plus tadalafil in pulmonary arterial hypertension. N Engl J Med 2015; 373: 834-844.

18 Humbert M, Sitbon O, Yaici A, et al. Survival in incident and prevalent cohorts of patients with pulmonary arterial hypertension. Eur Respir J 2010; 36: 549-555.

19 Sitbon O, Benza RL, Badesch DB, et al. Validation of two predictive models for survival in pulmonary arterial hypertension. Eur Respir J 2015; 46: 152-164.

20 Sitbon $\mathrm{O}$, Channick R, Chin KM, et al. Selexipag for the treatment of pulmonary arterial hypertension. $N$ Engl J Med 2015; 373: 2522-2533.

21 McLaughlin VV, Shillington A, Rich S. Survival in primary pulmonary hypertension: the impact of epoprostenol therapy. Circulation 2002; 106: 1477-1482.

22 Sitbon $\mathrm{O}$, Humbert $\mathrm{M}$, Nunes $\mathrm{H}$, et al. Long-term intravenous epoprostenol infusion in primary pulmonary hypertension: prognostic factors and survival. J Am Coll Cardiol 2002; 40: 780-788.

23 Wensel R, Opitz CF, Anker SD, et al. Assessment of survival in patients with primary pulmonary hypertension: importance of cardiopulmonary exercise testing. Circulation 2002; 106: 319-324. 
24 Nickel N, Golpon H, Greer M, et al. The prognostic impact of follow-up assessments in patients with idiopathic pulmonary arterial hypertension. Eur Respir J 2012; 39: 589-596.

25 McLaughlin VV, Gaine SP, Howard LS, et al. Treatment goals of pulmonary hypertension. J Am Coll Cardiol 2013; 62: D73-D81.

26 Kemp K, Savale L, O'Callaghan DS, et al. Usefulness of first-line combination therapy with epoprostenol and bosentan in pulmonary arterial hypertension: an observational study. J Heart Lung Transplant 2012; 31: 150-158.

27 Hassoun PM, Zamanian RT, Damico R, et al. Ambrisentan and tadalafil upfront combination therapy in scleroderma-associated PAH. Am J Respir Crit Care Med 2015; 192: 1102-1110.

28 Rich S. The effects of vasodilators in pulmonary hypertension. Pulmonary vascular or peripheral vascular? Circ Heart Fail 2009; 2: 145-150.

29 Galiè N, Ghofrani HA, Torbicki A, et al. Sildenafil citrate therapy for pulmonary arterial hypertension. $N$ Engl J Med 2005; 353: 2148-2157.

30 Galiè N, Brundage BH, Ghofrani HA, et al. Tadalafil therapy for pulmonary arterial hypertension. Circulation 2009; 119: 2894-2903.

31 Humbert M, Barst RJ, Robbins IM, et al. Combination of bosentan with epoprostenol in pulmonary arterial hypertension: BREATHE-2. Eur Respir J 2004; 24: 353-359.

32 Bachetti C, Manes A, Dardi F, et al. Comparison between initial combination therapy and initial monotherapy in pulmonary arterial hypertension: a single centre blinded evaluation of patients enrolled in the AMBITION study. Am J Respir Crit Care Med 2015; 191: A4779.

33 Bergot E, Sitbon O, Cottin V, et al. Current epoprostenol use in patients with severe idiopathic, heritable or anorexigen-associated pulmonary arterial hypertension: data from the French pulmonary hypertension registry. Int J Cardiol 2014; 172: 561-567.

34 Kylhammar D, Persson L, Hesselstrand R, et al. Prognosis and response to first-line single and combination therapy in pulmonary arterial hypertension. Scand Cardiovasc J 2014; 48: 223-233.

35 D'Alonzo GE, Barst RJ, Ayres SM, et al. Survival in patients with primary pulmonary hypertension: results from a national prospective registry. Ann Intern Med 1991; 115: 343-349.

36 Benza RL, Miller DP, Barst RJ, et al. An evaluation of long-term survival from time of diagnosis in pulmonary arterial hypertension from the REVEAL Registry. Chest 2012; 142: 448-456. 